\title{
Hipertensión renovascular secundaria a hipoplasia congénita de arterias renales en un paciente adulto joven. A propósito de un caso \\ Renovascular hypertension secondary to congenital hypoplasia of renal arteries in middle adult patient. About a case
}

\author{
Julián Darío Nañez Paz ${ }^{1, *}$, Juan David Orozco Burbano², Natalia Hernández Duque ${ }^{3}$ \\ ${ }^{1} \mathrm{MD}$ internista, Unidad de cuidado intensivo, Hospital Universitario San José, Popayán, Colombia \\ ${ }^{2} \mathrm{MD}$ general, Unidad de urgencias, Hospital Universitario San José, Popayán, Colombia \\ ${ }^{3}$ MD general, Unidad de urgencias, Hospital Universitario San José, Popayán, Colombia
}

\begin{abstract}
Resumen
La hipotrofia de aspecto tubular de las arterias renales constituye una rara entidad clínica asociada a la aparición de hipertensión renovascular. Su enfoque diagnóstico es complejo y exige la disponibilidad de angiografía para determinar las características propias del contorno de la vasculatura renal. El caso clínico considerado en este artículo ejemplifica el proceso diagnóstico de un paciente con esta particular situación.
\end{abstract}

Palabras clave: hipoplasia, arteria renal, hipertensión renovascular, insuficiencia renal aguda.

doi: http://dx.doi.org/10.22265/acnef.5.2.288

\begin{abstract}
The tubular hypotrophy of the renal arteries constitutes a rare clinical entity, that is associated with the appearance of renovascular hypertension. Its diagnostic approach is complex and requires the availability of angiography to determine the features of the renal vessels. The following case exemplifies the diagnostic process of a patient with this special clinical situation.
\end{abstract}

Key words: Hypoplasia, renal artery, renovascular hypertension, acute kidney injury.

doi: http://dx.doi.org/10.22265/acnef.5.2.288

Referenciar este artículo: Ñañez Paz JD, Orozco Burbano JD, Hernández Duque N. Hipertensión renovascular secundaria a hipoplasia congénita de arterias renales en un paciente adulto joven. A propósito de un caso. Rev. Colomb. Nefrol. 2018;5(1): 68-73. doi: http://dx.doi.org/10.22265/acnef.5.2.288

*Correspondencia: Julián Darío Ñañez, jnpaz@unicauca.edu.co

Recibido: 10-07-17 • Aceptado: 17-11-17 • Publicado en línea: 23-11-17 


\section{Introducción}

L a hipoplasia de arterias renales es una enfermedad rara. Se caracteriza por la hipotrofia de aspecto tubular de estas arterias; sus consecuencias clínicas se derivan de las repercusiones hemodinámicas intrarrenales. A pesar de esto, las descripciones de esta enfermedad en la literatura se limitan a algunos reportes de casos con compromiso principalmente de la aorta torácica y abdominal. A continuación, sin embargo, se describe el caso de un paciente joven con diagnóstico de hipoplasia de las arterias renales.

El paciente presenta un diagnóstico previo de hipertensión arterial esencial. A su ingreso, se documenta cuadro de falla renal crónica agudizada, sumado a cifras elevadas de presión arterial. A pesar del manejo ambulatorio antihipertensivo, este hallazgo no es explicado por el cuadro clínico por el cual consulta. A lo largo de su estadía hospitalaria y durante la realización de estudios paraclínicos se le diagnostica hipoplasia de las arterias renales.

\section{Presentación del caso clínico}

Paciente de 30 años de edad, sexo masculino, raza mestiza, oriundo de Neiva, residente en la ciudad de Popayán, estudiante de Ingeniería Civil. Tiene un antecedente importante de hipertensión arterial, diagnosticada en consulta externa hace dos años, sin estudios complementarios y manejada como hipertensión esencial con losartán $50 \mathrm{mg}$ cada doce horas, sin otros datos relevantes. También cuenta con historia de rinoplastia por trauma previo hace diez años. No refiere ningún otro antecedente patológico.

El paciente ingresa a consulta en el Hospital Universitario San José, por cuadro clínico de quince días de evolución, consistente en deposiciones diarreicas no disentéricas, asociadas a episodios de emesis de contenido alimentario, sin dolor abdominal, distermias u otro tipo de sintomatología. En el examen físico de ingreso, se encontró que el paciente estaba en regulares condiciones generales y con los siguientes signos vitales: presión arterial de 140/78 $\mathrm{mmHg}$, frecuencia cardiaca de 102 latidos por minuto, 21 respiraciones por minuto y temperatura de $37^{\circ} \mathrm{C}$. En la inspección general, llama la atención la marcada palidez conjuntival e ingurgitación yugular a $30^{\circ}$; a nivel pulmonar, estertores finos en ambas bases pulmonares sin otros sobreagregados; a nivel de miembros inferiores, se documentó edema grado II. No se evidenció ningún otro hallazgo positivo. Cabe aclarar, en todo caso, que se realizó una minuciosa exploración clínica.

Al ingreso se solicitaron paraclínicos, en los cuales se destacaron niveles marcadamente elevados de azoados con alteración electrolítica, dados por la presencia de hiperkalemia sin repercusión electrocardiográfica y síndrome anémico normocítico normocrómico. Por medio de un parcial de orina, se evidenció hipostenuria, proteinuria, hematuria y cilindros granulosos (Tabla 1).

Teniendo en cuenta la carencia de estudios previos y el antecedente de hipertensión, catalogada previamente como esencial, se plantea que, en el momento, cursa con una enfermedad renal crónica agudizada de origen prerrenal AKI-III secundario a su cuadro diarreico. No obstante, al considerar la edad y los paraclínicos de ingreso, se tuvieron en consideración otras patologías. Por consiguiente, se inicia tratamiento sindromático con amlodipino, esquema de líquidos controlados y medidas para control de hiperkalemia, como nebulizaciones de terbutalina, furosemida, gluconato de calcio y resinas de intercambio iónico.

A pesar del manejo instaurado, el paciente no presenta una evolución clínica satisfactoria, pues presentó posterior aparición de anuria, estado hipervolémico, acidosis metabólica, persistencia de hiperkalemia sin cambios electrocardiográficos y cifras tensionales con tendencia a elevación. Por este motivo, fue valorado por el servicio de nefrología, que inició terapia de reemplazo renal a las 48 horas de su ingreso, con mejoría del estado urémico e hipervolemia.

Posteriormente, se solicita ecografía renal con doppler de arteriales renales, donde se documenta como hallazgo importante: riñones con dimensiones disminuidas, presencia de atrofia renal bilateral simétrica con aumento de la ecogenicidad, compatible con nefropatía crónica sin dilatación del 
sistema colector, masas o cálculos. Se reporta en el estudio que tanto vasos venosos como arteriales intrarrenales izquierdos eran de aspecto normal, pero no es posible visualizar los derechos.

A continuación, se solicita perfil inmunológico y otros reactantes de fase aguda que resultaron negativos. Por esto, el grupo de nefrología del hospital decide realizar arteriografía renal, dado que no se dispone de angioresonancia en el momento, previa estabilización en su estado hidroelectrolítico y azoados, con protocolo de nefroprotección y posterior hemodiálisis. Este estudio determina la presencia de hipoplasia de arterias renales (ver Figuras 1 y 2).

Finalmente, al paciente se le define plan de egreso, dada la mejoría clínica y paraclínica en el estado de agudización de su falla renal, con diagnóstico de hipoplasia de arterias renales. Consecuentemente, se da plan de manejo externo con amlodipino 10 mg día y clonidina $150 \mathrm{mcg}$ cada doce horas, con recomendaciones generales y control ambulatorio estricto por nefrología para seguimiento, estudios de extensión y evaluación de necesidad de terapia de reemplazo renal.

Tabla 1. Paraclínicos de ingreso y egreso

\begin{tabular}{|c|c|c|c|}
\hline Examen de laboratorio & Ingreso & Egreso & Interpretación \\
\hline Leucocitos & 9.300 células $/ \mathrm{mm} 3$ & 7.800 células $/ \mathrm{mm} 3$ & Sin cambios: normales \\
\hline Neutrófilos & $7998 \mathrm{cel} / \mathrm{mm} 3$ & $5928 \mathrm{cel} / \mathrm{mm} 3$ & Sin cambios: normales \\
\hline Linfocitos & $744 \mathrm{cel} / \mathrm{mm} 3$ & $1248 \mathrm{cel} / \mathrm{mm} 3$ & Sin cambios: normales \\
\hline Hemoglobina & $12.1 \mathrm{~g} / \mathrm{dL}$ & 13.2 & Bajo \\
\hline Hematocrito & $36.9 \%$ & $38.7 \%$ & Bajo \\
\hline Plaquetas & $182.000 \times 103 / \mathrm{mm} 3$ & $184.000103 / \mathrm{mm} 3$ & Sin cambios: normales \\
\hline Tiempo de Protrombina & $10.1 \mathrm{~s}$. & $9.7 \mathrm{~s}$. & Sin cambios: normales \\
\hline Tiempo Parcial de Tromboplastina & $27 \mathrm{~s}$. & $26 \mathrm{~s}$. & Sin cambios: normales \\
\hline Proteína $\mathrm{C}$ reactiva & $1.1 \mathrm{mg} / \mathrm{dL}$ & - & Negativa \\
\hline Creatinina sérica & $10 \mathrm{mg} / \mathrm{dL}$ & $7.8 \mathrm{mg} / \mathrm{dL}$ & Elevada \\
\hline Nitrógeno ureico sérico & $69 \mathrm{mg} / \mathrm{dL}$ & $38 \mathrm{mg} / \mathrm{dL}$ & Elevado \\
\hline Sodio & $134 \mathrm{mEq} / \mathrm{L}$ & $139 \mathrm{mEq} / \mathrm{L}$ & Normal \\
\hline Potasio & $6.6 \mathrm{mEq} / \mathrm{L}$ & $3.7 \mathrm{mEq} / \mathrm{L}$ & $\begin{array}{c}\text { Hiperkalemia con posterior potasio } \\
\text { normal. }\end{array}$ \\
\hline Lactato sérico & 1.8 & - & Normal \\
\hline Proteinuria en 24 horas & $1712 \mathrm{mg} / 24$ horas & - & $\begin{array}{l}\text { Proteinuria presente rango no } \\
\text { nefrótico }\end{array}$ \\
\hline Factores del complemento $\quad$ C3-C4 & 160 y $37 \mathrm{mg} / \mathrm{dl}$ & - & Normales \\
\hline Anticuerpos Antinucleares (ANAs) & Negativos & - & Negativos \\
\hline Anticuerpos Anti DNA & Negativos & - & Negativos \\
\hline $\begin{array}{l}\text { anticuerpos anticitoplasma de neutrófilo } \\
\text { (ANCA IgG) }\end{array}$ & No detectado & - & Negativos \\
\hline $\begin{array}{l}\text { patrón de fluorescencia perinuclear } \\
\text { (P-ANCA) -citoplasmático (C-ANCA) }\end{array}$ & No detectado & - & Negativos \\
\hline Antitrombina III & $79 \%$ & - & $70-130 \%$ de inhibición \\
\hline Proteína C & $98 \%$ & - & $60-150 \%$ de inhibición \\
\hline Proteína S & $118 \%$ & - & $60-150 \%$ de inhibición \\
\hline Factor V Leiden & Negativos & - & Negativo \\
\hline Citoquímico de Orina & \begin{tabular}{l}
\multicolumn{1}{c}{ PH: 6, } \\
Proteínas: $100 \mathrm{mg} / \mathrm{dl}$ \\
Densidad: 1.005 \\
Glucosa: (-) \\
Cilindros: (-) \\
Bilirrubinas: (-) \\
Urobilinogeno: (-) \\
Nitritos: (-) \\
Hemoglobina: 80 \\
Leucocitos: 0-2 XC \\
Hematies: 6-8 XC \\
Células epiteliales: 0-1XC \\
Bacterias: Escasas \\
Cilindros granulosos: 1XC
\end{tabular} & & $\begin{array}{l}\text { Alterado: proteinuria-hipostenuria- } \\
\text { hematuria cilindros granulosos }\end{array}$ \\
\hline
\end{tabular}




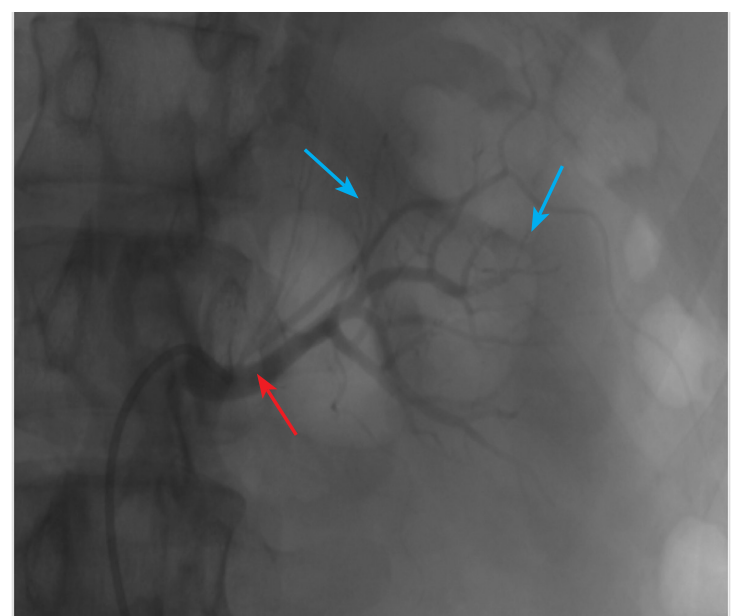

Figura 1

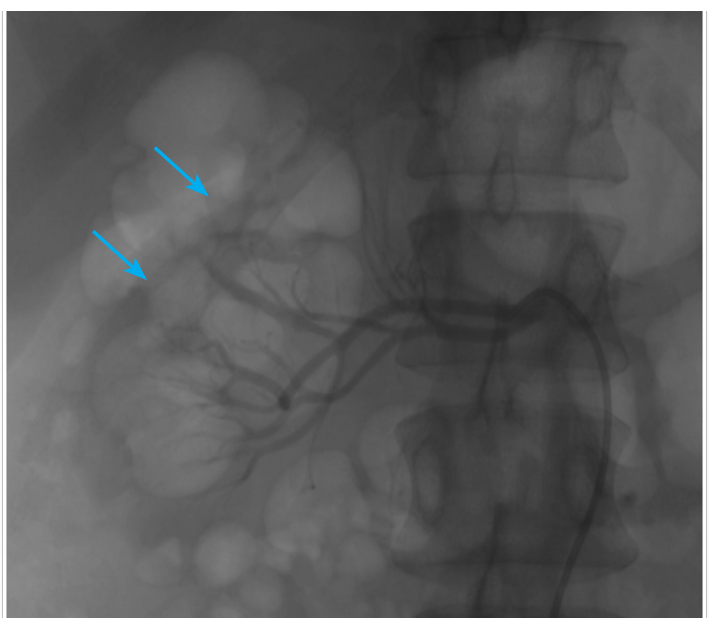

Figura 2

Figura 1 y 2. Arteriografía renal. Las arterias renales derecha e izquierda son de pequeño calibre con bifurcación en el tercio proximal y medio casi ostial con polares de muy pequeño calibre (flechas azules), visualizándose en fase nefrográfica retardada (flecha roja), sin presencia de dilataciones aneurismáticas, otras obstrucciones ni malformaciones arteriovenosa.

\section{Discusión}

La hipoplasia de arterias renales continúa siendo un problema clínico confuso, debido a la baja prevalencia de esta enfermedad. Esto ha restringido el desarrollo de una definición específica y un adecuado enfoque clínico. La patología hace parte de un gran número de condiciones incluidas en los síndromes CAKUT (congenital anomalies of the kidney and urinary tract), se caracteriza por un crecimiento subnormal del riñón que compromete, a su vez, la arteria renal y sus ramas, que son, además, hipoplásicas y comúnmente escleróticas ${ }^{1}$.

El término de hipoplasia renal congénita indica la presencia de un riñón de tamaño infantil con una función excretora preservada, a diferencia de las alteraciones funcionales relacionadas con las malformaciones vasculares propias de la hipoplasia de arterias renales ${ }^{2}$. Así, la hipoplasia de una arteria se encuentra definida como una estrechez regular, elongada y tubular de los segmentos de dicha estructura $^{3}$. Aunque se desconoce con claridad su fisiopatología, tanto la hipoplasia renal congénita como la hipoplasia de arterias renales tienen una fisiopatología en común, relacionada con defectos en la embriogénesis, que incluyen alteraciones en el desarrollo del blastema nefrogénico de ductos metanefrogénicos o deficiencias vasculares primarias.

Las arterias hipoplásicas y estenóticas tienen como manifestación principal la aparición de hipertensión renovascular. Esta situación puede ser explicada a través del fenómeno descrito por Goldblatt, en que, en modelos animales con un solo riñón, la oclusión de una de las tres ramas principales de las arterias renales se traducía en un incremento significativo de los niveles de renina y la aparición de hipertensión arterial secundaria ${ }^{4-5}$.

Como se ha mencionado, respecto a la frecuencia de esta condición cabe anotar que, por ser una patología poco usual, se disponen de escasos reportes de pacientes adultos en la literatura ${ }^{2} \mathrm{y}$, generalmente, se diagnostica en los primeros estadios de la vida, con periodos de latencia diferentes ${ }^{3-7}$.

Por lo tanto, no es infrecuente encontrar una asociación entre la hipoplasia de arterias renales concomitando con hipoplasia de la aorta y una frecuencia variable entre el 33 y el $81 \%{ }^{3}$. Sin embargo, ambas condiciones son en extremo raras e inusuales ${ }^{8}$. Como se dijo anteriormente, 
esta patología puede debutar clínicamente con deterioro renal agudo o presentarse como cuadros de hipertensión de difícil manejo en pacientes jóvenes. Por lo tanto, aunque sea infrecuente, se debe sospechar en pacientes con características como las mencionadas.

Considerando lo expuesto, es de vital importancia contar con paraclínicos e imágenes diagnósticas que permitan un adecuado enfoque y que no retarden el diagnóstico de esta patología. Por esto, la arteriografía es el estudio de elección, dado que permite definir si la hipoplasia es congénita o se trata de un caso de atrofia renal, teniendo en cuenta que, en la hipoplasia, la arteria renal se encuentra presente, aunque con un menor tamaño y longitud, como lo definen Love y colaboradores ${ }^{9}$. Con frecuencia, las características angiográficas más prominentes de esta patología consisten en la presencia de una arteria renal de menor tamaño desde su origen hasta la bifurcación, lo cual implica una estrechez con contornos tubulares sin estenosis focales. Asimismo, las arterias lobares y arcuatas se bifurcan de forma adecuada, pero se encuentran en menor tamaño ${ }^{2}$, a diferencia de la atrofia renal, en que el tamaño de la arteria se encuentra disminuido en su origen, pero sus ramas tienen apariencia irregular en los diferentes segmentos con estenosis focales.

Para finalizar, es importante insistir en que, entre las estrategiasdemanejo paraestacondición, se carece de guías clínicas o metaanálisis. Esta circunstancia puede ser atribuida a la escasa literatura presente, por lo que su enfoque terapéutico debe estar basado en la condición clínica del paciente y en el grado de afectación orgánica que presente. Como opción, se encuentra la nefrectomía en aquellos casos donde el compromiso es unilateral y se obtienen buenos resultados en objetivos como controlar las cifras tensionales y evitar el compromiso de otros órganos diana producto de la hipertensión arterial ${ }^{6,10}$.

\section{Conclusión}

La hipoplasia de arterias renales es una enfermedad poco frecuente y debe ser tenida en cuenta en el abordaje de pacientes con hipertensión arterial de causa secundaria. Con este artículo se hace énfasis en la importancia de abordar de manera integral a un paciente que debute con cuadro de hipertensión a temprana edad y sin antecedentes familiares de hipertensión. Con dicho ánimo, se describe el caso clínico de este paciente hipertenso sin estudios ambulatorios, cuyo cuadro de presentación llevó a que se descartaran causas comunes en nuestro medio. Dado que el hallazgo imagenológico fue la clave para llegar al diagnóstico, el hallazgo de esta patología, entre otras causas de hipertensión secundaria, exige un alto índice de sospecha, así como disponibilidad de apoyo diagnóstico con imágenes (angioresonancia o arteriografía, como en este caso), para prevenir posteriores complicaciones relacionadas con elevaciones sostenidas de la presión arterial.

\section{Conflicto de intereses}

Los autores declaran no tener conflicto de intereses en la realización de este artículo.

\section{Responsabilidades éticas}

\section{Protección de personas y animales}

Los autores declaran que para esta investigación no se han realizado experimentos en seres humanos ni en animales.

\section{Confidencialidad de los datos}

Los autores declaran que han seguido los protocolos de su centro de trabajo sobre la publicación de datos de pacientes.

\section{Derecho a la privacidad y consentimiento informado}

Los autores declaran que en este artículo no aparecen datos de pacientes.

\section{Contribución de los autores}

El caso fue identificado por el Dr. Juan David Orozco.

Estructuración y elaboración del cuerpo inicial del texto Dra. Natalia Hernández.

Asesor y estructuración final: Dr. Julián Darío Ñañez. 


\section{Referencias}

1. Ekstrom T. Renal Hypoplasia; a clinical study of 179 cases. Acta Chirurgica Scandinavica Supplementum. 1955;203:1-168.

2. Cha EM, Kandzari S, Khoury GH. Congenital renal hypoplasia: angiographic study. The American Journal of Roentgenology, Radium Therapy, and Nuclear Medicine. 1972;114(4):710-4. Doi: https://doi.org/10.2214/ajr.114.4.710

3. Odero A, Bozzani A, Arici V, Agozzino M. Hypoplasia and fibromuscular dysplasia of infrarenal abdominal aorta with downstream aneurysm: case report and review of the literature. Journal of Vascular Surgery. 2008;48(6):1589-92. Doi: https://doi.org/10.1016/j.jvs.2008.06.032

4. Brueggemeyer C, Farber MS, Ramirez G. Goldblatt phenomenon in a single kidney: the importance of subselective renal vein renins. Southern Medical Journal. 1984;77(10):1312-4. Doi: https://doi.org/10.1097/00007611-198410000-00026

5. Goldblatt H, Kahn JR, Bayless F, Simon MA. Studies on Experimental Hypertension : Xi. The Effect of Excision of the Carotid Sinuses on Experimental Hypertension Produced by Renal Ischemia. The Journal of Experimental Medicine. 1940;71(2):175-85. Doi: https://doi.org/10.1084/jem.71.2.175

6. Tozzi MC, Bruni L, Grisolia A, Lampariello S, Marcolongo P. [Hypoplasia of the renal artery as a cause of renovascular hypertension. A case report]. Minerva Pediatrica. 1993;45(1-2):35-9.

7. Arnold WC, Jimenez JF, Smith P, Norton JB, Redman JF. Renovascular hypertension in an infant with segmental renal artery stenosis and hypoplasia of the abdominal aorta. The Journal of Urology. 1983;130(1):127-8. Doi: https://doi.org/10.1016/S00225347(17)50991-X

8. Lin YJ, Hwang B, Lee PC, Yang LY, Meng CC. Mid-aortic syndrome: a case report and review of the literature. International Journal of Cardiology. 2008;123(3):348-52. Doi: https://doi.org/10.1016/j.ijcard.2006.11.167

9. Love L, Des Rosiers RJ. Angiography of renal agenesis and dysgenesis. The American Journal of Roentgenology, Radium Therapy, and Nuclear Medicine. 1966;98(1):137-42. Doi: https://doi.org/10.2214/ajr.98.1.137

10. Burkland CE, Goodwin WE, Leadbetter WF. The cure of hypertension by nephrectomy; a ten-year follow-up of a case. Surgery. 1950;28(1):67-70. 